\title{
Condições norteadoras para caracterização de incapacidade laborativa por epilepsia
}

\author{
Specific conditions to help orient experts to characterize \\ work disability caused by epilepsy
}

\author{
Ludmila Candida de Braga'; ${ }^{1}$ Victor Alexandre Percinio Gianvecchio ${ }^{2}$
}

D0I : http://dx.doi.org/10.11606/issn.2317-2770.v19i2p67-77

Braga LC; Gianvecchio VAP. Condições norteadoras para caracterização de incapacidade laborativa por epilepsia. Saúde, Ética \& Justiça. 2014;19(2):67-77.

RESUMO: INTRODUÇÃO: A epilepsia é problema de Saúde Pública e importante causa de incapacidade laborativa no Brasil e no mundo, sendo condição de interesse no contexto médico-pericial. OBJETIVO: Descrever condições norteadoras para caracterização de incapacidade laborativa por epilepsia. MATERIAL E MÉTODO: Revisão de literatura. RESULTADOS E DISCUSSÃO: O diagnóstico de epilepsia por si só não é sinônimo de incapacidade. Grande parte dos casos tem bom prognóstico com controle do quadro e preservação da capacidade laborativa. Entretanto, incapacidade laborativa pode advir dessa condição e, em geral, relaciona-se às suas repercussões somáticas ou psíquicas, comorbidades psiquiátricas, efeitos colaterais das drogas antiepiléticas e quando se verifica risco à saúde do trabalhador e/ou a terceiros. São fatores que favorecem a presença de incapacidade: crises frequentes, crises com perda de consciência, crises de início súbito, crises sem fatores desencadeantes, crises em vigília, não adesão ao tratamento, alcoolismo ou uso de outras drogas, epilepsia de causas estruturais, metabólicas ou desconhecidas, epilepsia refratária a tratamento. Outros aspectos podem sinalizar gravidade da doença, mas sua utilização na avaliação pericial deve ser relativizada: acompanhamento em serviço de referência, inclusão em programas de dispensação de medicamentos especiais, realização de exames complementares diferenciados, indicação de avaliação neuropsicológica, politerapia em altas doses, interações frequentes, eletroencefalograma persistentemente alterado, indicação de tratamento cirúrgico. Todos os medicamentos analisados nesta revisão podem desencadear efeitos potencialmente incapacitantes, com destaque para os relativos às funções cognitivas e comportamentais. Os resultados de exames complementares devem ser analisados à luz da clínica do periciando e não há achados ou resultados específicos que sejam sinônimo de incapacidade laborativa. Os autores tendem a caracterizar incompatibilidade entre algumas ocupações ou situações específicas no trabalho e epilepsia, mesmo quando esta se encontra controlada. São elas: profissões que envolvam atividades com riscos para si e para terceiros, tais quais piloto de avião, maquinista de trem, bombeiros, policiais, operadores de instrumentos e máquinas perigosas, vigias solitários, instrutor de natação e salva-vidas, babás, profissionais de enfermagem, cirurgiões, motoristas profissionais, militares, pessoas responsáveis por prestar cuidados a indivíduos vulneráveis, trabalho em altura ou com uso de escadas, próximo de água, com exposição a alta voltagem e circuitos elétricos abertos, próximo ou sobre veículos em movimento, subterrâneo, em contato com fogo, fornos e fogões. CONCLUSÕES: Existem pontos norteadores para caracterização da incapacidade laborativa por epilepsia. Preparo técnico, avaliação individualizada, atenta e detalhada pelo perito favorecem a produção de prova técnica fortemente sustentada por conhecimento científico, alcançando-se assim o objetivo da perícia médica que é se fazer justiça.

DESCRITORES: Pessoas com deficiência; Epilepsia; Avaliação da capacidade de trabalho; Prova pericial.

\footnotetext{
1. Médica, Doutora em Saúde Coletiva, Pós-graduada em Perícias Médicas, Médica Coordenadora Regional da Coordenadoria de Saúde e Segurança do Trabalhador e Sustentabilidade Ambiental da Universidade Estadual Paulista "Júlio de Mesquita Filho".

2. Médico, Perito da Vara de acidentes do Trabalho da Comarca de São Paulo, Vice-coordenador do Curso de Especialização em Perícias Médicas da Faculdade de Ciências Médicas da Santa Casa de São Paulo, e-mail: victorgianvecchio@hotmail.com

Endereço para correspondência: Ludmila Candida de Braga - e-mail: ludbra@fmb.unesp.br
} 


\section{INTRODUÇÃO}

Segundo Fischer et al. $(2005)^{I}$,

"epilepsia é o nome de uma desordem cerebral caracterizada predominantemente por interrupções recorrentes e imprevisiveis da atividade cerebral normal, chamadas de crises epiléticas. Epilepsia não é uma entidade nosológica especifica mas sim uma variedade de desordens refletindo disfunção cerebral que pode resultar de diferentes causas" e crise epilética é a "ocorrência transitória de sinais elou sintomas devidos a atividade anormal excessiva ou sincrona cerebral".

As causas são variáveis e envolvem danos cerebrais devidos a infecções, doenças parasitárias tais como neurocisticercose, trauma craniano, uso de drogas ilícitas, acidente vascular cerebral, tumores, malformações cerebrais ou vasculares e, em alguns casos, a causa é desconhecida ${ }^{2,3}$.

A apresentação clínica é bastante diversificada e correlacionada à área cortical afetada, com crises, que são eventos de curta duração, caracterizadas por alterações sensoriais, motoras ou outras, tais como atividade motora involuntária, manifestações autonômicas, liberação esfincteriana, experiências subjetivas, sensação de medo, parestesias, alucinações olfativas, gustativas, auditivas e visuais, déjà $v u$, jamais $v u$, pensamento forçado, vertigem, desequilíbrio, perda da consciência, entre outros ${ }^{2}$.

Segundo relatório da comissão de classificação da Liga Internacional Contra Epilepsia (ILAE) traduzido para o português ${ }^{4}$, a epilepsia pode ser classificada, com base em sua etiologia, em:

(a) Epilepsia genética: resultado direto de um defeito genético conhecido ou presumido em que as crises são o principal sintoma.

(b) Epilepsia estrutural/metabólica: há uma condição distinta ou estrutural ou metabólica ou outra doença que demonstrou estar associada a um substancial aumento do risco de desenvolver epilepsias.

(c) Epilepsia de causa desconhecida: natureza desconhecida.

O mesmo relatório apresenta a classificação das crises epiléticas. As crises epiléticas generalizadas são aquelas "originárias em algum ponto dentro de, e rapidamente envolvendo, redes distribuídas bilateralmente" e são subclassificadas em: tônicoclônica, ausência, mioclônica, clônica, tônica, atônica. Já as focais são aquelas "originárias dentro das redes limitadas a um hemisfério". Existem também as crises epiléticas classificadas como desconhecidas, que incluem os espasmos epiléticos ${ }^{4}$.

Ainda no que se refere às crises epiléticas, verifica-se que sua apresentação se dá com diferentes graus de envolvimento muscular. Quando sua manifestação se dá predominantemente por fenômeno motor, são denominadas convulsões e, quando não, são denominadas não-convulsivas ${ }^{3}$.

$\mathrm{Na}$ sua grande maioria, os casos de epilepsia podem ser controlados por meio de tratamento com drogas antiepiléticas a serem utilizadas regularmente $e^{2,5}$.

A meta do tratamento da epilepsia é alcançar o completo controle das crises com a carga mínima possível de efeitos colaterais dos medicamentos ${ }^{6}$.

Entretanto, as taxas de sucesso de tratamento são variáveis. Sander \& Sillanpää $(1998)^{7}$ apud WHO (2000), constataram que mais de $70 \%$ dos indivíduos epiléticos recentemente diagnosticados alcançam controle das crises quando tratados regularmente com drogas antiepiléticas. E, entre os $30 \%$ que permanecem com crises, grande parte tem a frequência das mesmas consideravelmente reduzida com a terapia.

O mesmo estudo mostrou que cerca de 60 a $70 \%$ dos sujeitos que permanecem por longos períodos sem crises podem ter sua medicação suspensa sem manifestar novas crises, se estiverem sob seguimento médico ${ }^{7}$.

Os indivíduos epiléticos podem ser agrupados em três categorias com relação ao controle do quadro: remissão com tratamento, remissão espontânea e permanência das crises apesar do tratamento ${ }^{2}$.

Entre os que mantem-se com crises apesar do tratamento medicamentoso, estão os casos enquadrados como intratáveis. Nesta situação, o controle pode ser alcançado por meio de tratamento neurocirúrgico ${ }^{2}$.

Indivíduos com epilepsia ativa são aqueles que, nos últimos doze meses, foram acometidos por, pelo menos, duas crises. Somente após um período de cinco anos sem tratamento e sem crises, uma pessoa é considerada sem epilepsia ${ }^{2}$.

O tratamento do indivíduo epilético pode contemplar outras abordagens não medicamentosas ou não cirúrgicas. É sabido que, em algumas situações, dieta específica pode contribuir para o controle das crises ${ }^{8}$.

Além disso, intervenções e apoio psicológico e social podem beneficiar o tratamento de indivíduos epiléticos, dadas as comorbidades e implicações psíquicas e sociais que os acometem ${ }^{2}$.

Trata-se de condição de grande magnitude, havendo estimativa de que 50 milhões de pessoas sejam por ela atingidas hoje no mundo, dos quais 40 milhões em países em desenvolvimento ${ }^{2}$.

Estudos epidemiológicos revelam prevalências que variam de 1,5 a 57 casos/1000 habitantes ${ }^{9,10}$.

A incidência em países industrializados varia de 40 a 70 casos/100000 habitantes/ano, enquanto, nos países em desenvolvimento, varia de 100 a 190 casos/100000 habitantes/ano ${ }^{11}$. 
Somam-se à magnitude do problema, suas repercussões individuais, familiares e sociais, tais como danos físicos, intimamente relacionadas à imprevisibilidade das crises epiléticas, alterações psíquicas em decorrência da falta de controle sobre as crises e estigma e exclusão social, com prejuízos à escolarização, relacionamentos afetivos e acesso e manutenção do emprego ${ }^{11}$. Sendo assim, é possível caracterizar a epilepsia como um problema de Saúde Pública.

Em artigo de revisão, Gomes (2009) ${ }^{12}$, verifica que, entre 21 grupos, as doenças do sistema nervoso ocupam a oitava posição na concessão de benefícios previdenciários do tipo auxílio-doença, segundo estatísticas o Instituto Nacional de Seguro Social do Brasil. Neste grupo, a epilepsia é o agravo mais frequente como causa de incapacidade laborativa.

Segundo Miziara et al. (2011) $)^{13}$, estatísticas do Instituto Nacional de Seguro Social revelam, no período de 2005 a 2010, a concessão de 46.151 benefícios previdenciários do tipo auxílio-doença por epilepsia e estado de mal epilético, com tendência de queda no número de concessões ao longo dos anos.

Em inquérito populacional conduzido por Gannon $\&$ Nolan $(2004)^{14}$ na Irlanda, verificou-se que, entre 287 mil entrevistados na faixa etária de 15 a 64 anos, quase $11 \%$ referiram apresentar problemas de saúde de longa duração (superior a seis meses) ou incapacidade. Entre eles, 2,2\% mencionaram a epilepsia como causa da incapacidade/problema de saúde de longa duração.

Trevisol-Bittencourt et al. (2001) $)^{15}$, em estudo que avaliou as principais causas de encaminhamento por médicos peritos da Previdência Social de Santa Catarina a perito especialista em neurologia, verificaram que a epilepsia era o principal motivo de encaminhamento, respondendo por $32,4 \%$ dos casos.

Para Gomes $(2009)^{12}$, a epilepsia pode levar à incapacidade laborativa, quando restringir o exercício da atividade profissional, bem como quando interferir negativamente na capacidade de ocupação e integração profissional, além das situações em que houver risco de vida para si ou terceiro ou risco de agravamento da doença pela permanência no trabalho.

A incapacidade laborativa relacionada à epilepsia pode advir do próprio agravo, das comorbidades psíquicas ou somáticas ou dos efeitos colaterais do tratamento ${ }^{12,13,16,17,18}$.

Outros estudos revelam maiores taxas de desemprego entre epiléticos que na população geral e associam este achado a aspectos do agravo e de ordem social, tais como ocorrência de crise epilética no ambiente de trabalho e baixo nível de escolaridade ${ }^{19}$.

Segundo Miziara et al. $(2011)^{13}$, em artigo de revisão, existem poucos estudos que tratam exclusivamente da questão médico-legal relacionada à epilepsia. Segundo os autores,

$$
\begin{aligned}
& \text { "a simples presença do diagnóstico de epilepsia não } \\
& \text { implica em incapacidade para o trabalho, não sendo } \\
& \text { possivel prever a ocorrência de crise no trabalho nem o } \\
& \text { aumento de risco à saúde do trabalhador e de terceiros } \\
& \text { em decorrência dela". }
\end{aligned}
$$

Em concordância com a conclusão de que o diagnóstico de epilepsia não implica necessariamente incapacidade laborativa, Kwan \& Sander $(2004)^{5}$ afirmam, em artigo de revisão, que mais de $70 \%$ dos portadores de epilepsia diagnosticados recentemente tem o quadro controlado com tratamento medicamentoso.

Miziara et al. (2011) $)^{13}$ apontam que " $a$ avaliação médico-pericial deve abordar as condições médicas (manifestações clínicas, exames físico geral e neurológico e resultados de exames complementares) e as ocupacionais do periciando" e enfatizam a importância das informações contidas em atestado de médico assistente para conclusão de parecer médico-pericial, o que, por si só, revela a dificuldade que se apresenta ao médico perito no momento da avaliação, tendo em vista que muitas vezes tais documentos trazem informações escassas ou que não refletem a realidade do periciando.

Considerando a magnitude da epilepsia no mundo e em países em desenvolvimento como o Brasil, suas profundas repercussões negativas sobre a qualidade de vida e capacidade laborativa dos indivíduos, a elevada frequência com que sujeitos com epilepsia se apresentam aos serviços de perícia médica pleiteando a constatação de incapacidade laborativa e as dificuldades vivenciadas pelo médico perito no momento de emitir sua decisão, justifica-se a realização do presente estudo, de modo a revisar e organizar achados de literatura sob a forma de material de apoio à tomada de decisão médico-pericial.

\section{OBJETIVOS}

Identificar condições norteadoras para caracterização de incapacidade laborativa por epilepsia.

\section{MATERIAL E MÉTODO}

Trata-se de estudo do tipo revisão de literatura.

A pesquisa foi realizada nas bases de dados disponíveis nos seguintes sites: National Center for Biotecnology Information, Scielo e Google acadêmico. Além disso, foram utilizados livros e material didático de curso sobre perícia em saúde promovido por instituição pública de ensino superior do Estado de São Paulo em 2011.

Foram utilizados os seguintes descritores para revisão de literatura: epilepsia, incapacidade, incapacidade laborativa, trabalho, tratamento, em português e inglês. 
Os critérios de inclusão dos artigos foram: artigos de livre acesso e disponíveis na íntegra e que, de fato, abordassem aspectos médico-legais. Quando, durante a leitura de um artigo, foi identificada outra referência de interesse, os autores buscaram intencionalmente a referência original.

Para fins de descrição de efeitos colaterais do tratamento medicamentoso da epilepsia, foram utilizadas monografias de medicamentos disponíveis em sites especializados.

Para fins de organização do texto, optou-se por descrever os achados considerando-se os seguintes itens:

- aspectos e repercussões físicas e psíquicas do agravo

- efeitos adversos das drogas antiepiléticas mais comumente utilizadas

- aspectos de exames complementares

- especificidades de determinadas ocupações ou condições de trabalho

\section{RESULTADOS}

O diagnóstico de epilepsia não é por si só sinônimo de incapacidade laborativa ${ }^{12,13,20,21}$. E embora, na maioria dos casos de epilepsia haja sucesso no controle da doença e bom prognóstico com potencial de preservação da capacidade laborativa a despeito da cronicidade do quadro, o agravo pode gerar incapacidade. Por isso, o estabelecimento de critérios ou, ao menos, norteadores de incapacidade laborativa por epilepsia é de interesse à prática pericial.

Antes de tratar especificamente da incapacidade laborativa por epilepsia, há que se deter sobre o paradigma de incapacidade a ser adotado, pois ele é definidor de que aspectos devem ser considerados na construção do parecer médico-pericial.

O paradigma biomédico da incapacidade mostrase insuficiente para responder às demandas impostas às sociedades modernas e, segundo Loisel et al. $(2005)^{22}$, "a incapacidade para o trabalho, na maioria das vezes, é o resultado de ações e interações inapropriadas entre os vários sistemas e um envolvimento descoordenado dos atores no processo de afastamento de cada trabalhador". Para o autor, a incapacidade é fruto de inadequada interação entre as características do sistema de saúde, do sistema de seguridade social, dos empregadores, das condições de trabalho, dos trabalhadores, entre outros, ou seja, é socialmente produzida.

Tal paradigma deve ser observado não só na elaboração de políticas públicas de prevenção da incapacidade, mas também no momento específico do exame pericial.

Em se tratando da incapacidade por epilepsia, é importante que a avaliação pericial seja ampliada para além dos aspectos biomédicos da doença, pois diversos fatores podem interferir na determinação da capacidade laborativa dos indivíduos, sejam eles: repercussões sobre a saúde física e mental do sujeito, possibilidade de deterioração das relações pessoais, familiares, sociais, dificuldades no processo de escolarização, problemas na inserção e permanência no mercado de trabalho, preconceito, estigma, cobertura e eficiência dos sistemas de saúde, eficiência dos programas de reabilitação profissional, entre outros.

Para conduzir essa avaliação ampliada, recomenda-se que o médico perito lance mão de todas as ferramentas a seu alcance, tais como: anamnese detalhada, entrevista com familiares ou acompanhantes, análise dos documentos médicos apresentados, exame clínico apurado (embora a grande maioria dos epiléticos apresente exame físico dentro da normalidade) e, em caso de dúvidas ou incoerência de informações, é recomendável a solicitação de prontuário médico ${ }^{20}$.

A seguir serão abordados possíveis critérios e norteadores para caracterização ou exclusão de incapacidade laborativa por epilepsia.

\section{Manifestações clínicas, comorbidades e avaliação de incapacidade por epilepsia}

As manifestações clínicas da epilepsia podem implicar em incapacidade laborativa.

Lehman, citado Gomes (2009) ${ }^{12}$, indica alguns fatores que podem alterar o ajuste socioprofissional do indivíduo com epilepsia. Seriam fatores favoráveis ao ajuste:

- crises infrequentes

- crises durante o período de ajuste de medicamentos

- crises parciais motoras que não interferem com a consciência e/ou controle motor

- crises com aura consistente e prolongada

- crises com padrão noturno

- crises secundárias a estados agudos metabólicos ou tóxicos improváveis de recorrer

- crises por privação de sono

- crises relacionadas a doenças agudas reversíveis

Cabe destacar que os autores não especificam numericamente o significado de crises infrequentes.

Seriam fatores desfavoráveis ao ajuste:

- não adesão ao tratamento

- alcoolismo ou abuso de drogas nos últimos três meses

- número aumentado de crises no último ano

- lesão cerebral estrutural

- condição metabólica ou funcional cerebral não corrigível 
Por isso, a história clínica e análise documental são de grande valor pericial, revelando informações sobre frequência, duração, tipo, horário de ocorrência das crises epiléticas, que são parâmetros definidores de incapacidade laborativa.

Também podem trazer ao conhecimento do perito outros critérios indiretos e sugestivos de gravidade da doença, tais quais: acompanhamento em serviços de referência, inclusão em programas de dispensação de medicamentos especiais, realização mais frequente de exames complementares diferenciados, história de avaliação neuropsicológica, politerapia em doses elevadas, internações frequentes, eletroencefalograma com alterações epileptiformes persistentes, deterioração psíquica ou neurológica, transtorno mental grave, indicação ou discussão sobre possibilidade de tratamento cirúrgico ${ }^{20}$.

A epilepsia tem como complicações: estado de mal epilético, traumatismos, morte súbita, comorbidade psiquiátrica $^{3}$. Embora não sejam previsíveis, sua presença ou risco de ocorrência devem ser considerados na avaliação pericial em associação ao contexto geral e ocupacional do periciando.

Especificamente no que se refere à comorbidade entre epilepsia e transtornos mentais e do comportamento, vários estudos indicam associação entre as referidas condições $^{3,24,25,26}$. A incapacidade laborativa relacionada à epilepsia também pode decorrer da presença de transtornos mentais e do comportamento, devendo o médico perito estar atento a essa possibilidade ${ }^{13}$.

A quase totalidade de artigos que tratam da incapacidade laborativa por epilepsia selecionados e descritos no presente estudo não apresentou critérios fechados para caracterização de incapacidade, mas, sim, descreveu sinais orientadores da avaliação. Apenas o documento que apresenta as diretrizes de apoio à tomada de decisão médico-pericial do Instituto Nacional de Seguro Social sugere parâmetros mais estanques para a caracterização de incapacidade ${ }^{20}$, conforme o quadro a seguir:

QUADRO 1 - Conduta médico-pericial nas epilepsias, segundo INSS (2009)

\begin{tabular}{|l|l|}
\hline Ausência de incapacidade & $\begin{array}{l}\text { Crises bizarras, prolongadas e polimórficas, com manifestações clínicas que não } \\
\text { se enquadram nas síndromes epiléticas, em subdose de medicamento e EEG } \\
\text { persistentemente normal. }\end{array}$ \\
\hline Data para cessação de benefício & $\begin{array}{l}\text { Epilepsia recém diagnosticada em fase de investigação e adaptação de uso de } \\
\text { drogas antiepiléticas: concessão de 30 dias e, se ainda instável, prolongamento } \\
\text { de prazo favorável até 60 dias. } \\
\text { Epilepsia sintomática (malformação arteriovenosa, processo expansivo, trauma } \\
\text { crânio-encefálico): considerar doença de base. }\end{array}$ \\
\hline Reabilitação profissional & $\begin{array}{l}\text { Epilepsia bem definida (lembrar dos diagnósticos diferenciais como síncopes, } \\
\text { síndromes vertiginosas, etc) no contexto de profissões de risco (motorista, ope- } \\
\text { rador de máquinas pesadas, altura, etc) }\end{array}$ \\
\hline Revisão em dois anos & $\begin{array}{l}\text { Epilepsia refratária, bem documentada, em politerapia em altas doses (conside- } \\
\text { rar sinais clínicos de intoxicação e/ou níveis séricos das drogas antiepiléticas) } \\
\text { Casos elegíveis para cirurgia }\end{array}$ \\
\hline Limite indefinido & $\begin{array}{l}\text { Epilepsia refratária de longa data, em acompanhamentos em centros terciários, } \\
\text { associada à comorbidade psiquiátrica grave e/ou EEG persistentemente altera- } \\
\text { do, e/ou má resposta cirúrgica. }\end{array}$ \\
\hline
\end{tabular}

Fonte: Instituto Nacional de Seguro Social

\section{Características do tratamento, efeitos adversos das drogas antiepiléticas mais comumente utilizadas e avaliação de incapacidade por epilepsia}

Segundo WANNMACHER $(2008)^{27}$, o objetivo do tratamento do indivíduo com epilepsia é alcançar "melhor qualidade de vida para o paciente, com melhor controle das crises e o mínimo de efeitos adversos". Como a maioria dos indivíduos com epilepsia não obterá cura, os medicamentos antiepiléticos são prescritos com objetivo de se evitar a recorrência de crises.
Ainda segundo a autora ${ }^{27}$,"o passo mais importante para o sucesso terapêutico é a correta identificação de tipo de crise e síndrome epilética, já que os mecanismos de geração e propagação das crises são diferentes, e os vários anticonvulsivantes agem por diversos mecanismos que podem ou não ser favoráveis a cada síndrome”.

A escolha do medicamento a ser prescrito deve ser baseada em: tipo de crise/síndrome epilética, eficácia do medicamento, efeitos adversos do medicamento, tolerabilidade individual, facilidade de administração e custo de tratamento ${ }^{27}$. 
Preferencialmente, o tratamento deve ser iniciado com uma única droga, pois a "monoterapia reduz riscos de toxicidade e teratogenicidade, elimina problemas de interação framacológica, melhora a adesão dos pacientes e simplifica a avaliação da resposta terapêutica"27.

Contudo, em caso de insucesso com o tratamento inicial, não há consenso sobre a conduta a ser adotada, podendo haver indicação de aumento da dose da primeira droga até o aparecimento de efeitos colaterais limitantes, substituição por outra droga ainda em monoterapia ou mesmo a associação de outros fármacos ${ }^{27,28}$.

WANNMACHER $(2008)^{27}$ recomenda que, por ocasião da necessidade de associação de outras drogas, sua escolha seja orientada pelos seguintes aspectos: (i) associar drogas com diferentes mecanismos de ação, (ii) associar drogas com diferente escopo de efeitos colaterais e (iii) não associar drogas com alta capacidade de induzir ou inibir as enzimas hepáticas.

Além da adequada seleção da droga antiepilética a ser utilizada, outros fatores podem contribuir para sucesso e fracasso no controle das crises epiléticas. ANGALAKUDITI \& ANGALAKUDITI (2011 $)^{6}$ apontam que a qualidade da assistência ao portador de epilepsia pode diferir entre os países estudados em decorrência de características dos sistemas de saúde. Outros estudos revelam ocorrência elevada de indivíduos submetidos a tratamento inadequado e maior dificuldade de controle da doença em países em desenvolvimento ${ }^{2}$.

Tais achados têm implicação no contexto médico-pericial brasileiro em que, apesar dos avanços farmacológicos, peritos frequentemente se deparam com indivíduos com dificuldade de acesso ao sistema de saúde e ao tratamento preconizado, o que, além dos impactos peculiares ao tratamento, pode ser mais um fator que acarreta prejuízos à capacidade laborativa do periciando.

Em artigo de revisão, ANGALAKUDITI \& ANGALAKUDITI (2011) $)^{6}$ identificaram estudos acerca do tratamento da epilepsia, que totalizaram 12.317 pacientes com crises parciais ou generalizadas na Argentina, Brasil, China, República Tcheca, Índia, Coréia, Polônia, Rússia e Taiwan.

$\mathrm{Na}$ investigação $^{6}$, verificaram que dezessete diferentes drogas antiepiléticas foram utilizadas, conforme o que se segue: fenobarbital $(21,7 \%)$, valproato de sódio $(17,5 \%)$, tiagabina $(16,4 \%)$, oxcarbazepina $(8,3 \%)$, levetiracetam $(8,1 \%)$, topiramato $(7,2 \%)$, acetato de eslicarbazepine $(5,8 \%)$, lacosamida $(3,9 \%)$, lamotrigina $(2,1 \%)$, pregabalim $(1,9 \%)$, clobazam (1,6\%), zonisamida, primidona, clonazepam, fenitoína, gabapentina e carbamazepina (menos de $1 \%$ cada).

Em todos os estudos revisados, ficou demonstrada a redução da frequência e/ou severidade das crises e/ou controle total das crises, revelando a importância das drogas antiepiléticas no tratamento da patologia ${ }^{6}$.
Segundo o INSS (2009) ${ }^{20}$, as drogas antiepiléticas mais comumente utilizadas no país são: carbamazepina, fenobarbital, fenitoína, valproato, lamotrigina e topiramato.

Aldenkamp $(2001)^{16}$, em artigo de revisão, destaca como novas drogas antiepiléticas: oxcarbazepina, lamotrigina, gabapentina, vigabatrina, tiagabina, topiramato, levotiracetam e rufinamida.

São descritos a seguir os efeitos colaterais de cada uma das drogas mencionadas por INSS (2009) $)^{20} \mathrm{e}$ Aldenkamp (2001) ${ }^{16}$

1. CARBAMAZEPINA: náuseas, vômitos, sonolência, vertigens, cefaleia, ataxia, diplopia, síndrome de Stevens-Johnson, necrose epidérmica tópica, hiponatremia, diarréia, diaforese, síndrome de secreção inapropriada de HAD, discrasias sanguíneas (anemia aplástica e agranulocitose), hepatotoxicidade, anormalidades cardíacas, insuficiência renal, eosinofilia pulmonar e erupção cutânea, pancitopenia, trombocitopenia, leucopenia, neutropenia, eosinofilia, anemia hemolítica, arritmia cardíaca, falência cardíaca, cefaleia, confusão mental, depressão, agitação, psicose, mania, neurite periférica, hipotiroidismo, porfiria, hipertermia, ganho de peso, pancreatite, visão turva, retinopatia, acne, eritema multiforme, alopecia, osteomalácia e hipersensibilidade ${ }^{29}$.

2. FENITOÍNA: ataxia, fadiga, diplopia, vertigem, nistagmo, visão borrada, disfasia, tremores, hiperglicemia, depleção de ácido fólico, osteomalácia, hiperplasia gengival, hipertricose, rashs cutâneos, monoplegia ou hemiplegia reversíveis, parkisonismo reversível, linfoadenopatia, discrasias sanguíneas, síndrome de Stevens-Johnson, discinesia, hepatite crônica, pneumonia intersticial ${ }^{29}$.

3. FENOBARBITAL: tontura, sedação, depressão, transtornos comportamentais, prejuízo cognitivo e da concentração ${ }^{28}$.

4. GABAPENTINA: aumento do apetite, ganho de peso, tontura, ataxia, nistagmo, cefaleia, tremor, fadiga, diplopia, náusea ${ }^{28}$.

5. LAMOTRIGINA: rash cutâneo, cefaleia, diplopia, náusea, tontura, ataxia, tremor, astenia e ansiedade ${ }^{28}$.

6. LEVETIRACETAM: infecção, nasofaringite, trombocitopenia, anorexia, aumento de peso, agitação, depressão, labilidade emocional, variações do humor, hostilidade/agressividade, insônia, nervosismo/ irritabilidade, alterações da personalidade, perturbações do pensamento, sonolência, diplopia, visão desfocada, vertigem, aumento da tosse, dor abdominal, diarreia, dispepsia, náusea, vômitos, erupções cutâneas, eczema, 
prurido, mialgia, astenia, ferimento acidental. O fármaco não foi aprovado pela ANVISA e não é comercializado no Brasil ${ }^{30}$.

7. OXCARBAZEPINA: síndrome de StevensJohnson, necrose tóxica epidermal, sonolência, cefaleia, tontura, diplopia, náusea, vômito, fadiga, astenia, rash cutâneo, alopecia, acne, diarreia, constipação, dor abdominal, vertigem, ataxia, tremor, nistagmo, concentração prejudicada, amnésia, confusão, depressão, apatia, agitação, instabilidade emocional e hiponatremia ${ }^{31}$.

8. RUFINAMIDA: pneumonia, gripe, nasofaringite, otite, sinusite, rinite, anorexia, diminuição do apetite, sonolência, cefaleia, tontura, estado de mal epilético, convulsões, alterações da coordenação, nistagmo, tremor, diplopia, visão turva, vertigens, epistaxe, dor abdominal, constipação, náuseas, vômitos, dispepsia, diarreia, aumento das enzimas hepáticas, acne, rash cutâneo, dorsalgia, oligomenorreia, ataxia, fadiga, diminuição do peso, perturbações no andar suscetíveis de aumentar a ocorrência de quedas acidentais, febre e erupção cutânea associados ao envolvimento de um outro sistema de órgãos, linfadenopatia, hematúria, ideação suicida e visão turva ${ }^{32,33}$.

9. TIAGABINA: sonolência, vertigem, dificuldade de concentração, dispepsia, ansiedade, mioclonias, insônia, depressão, tremor, psicose, exantema ${ }^{34}$.

10. TOPIRAMATO: sonolência, anorexia, fadiga, nervosismo, pensamento lento, dificuldade de encontrar palavras, dificuldade de concentração, perda de peso, parestesias, dores abdominais, acidose metabólica, nefrolitíase, miopia e glaucoma de ângulo fechado ${ }^{28}$.

11. VALPROATO DE SÓDIO OU ÁCIDO VALPRÓICO: sedação, tontura, cefaleia, náuseas, vômitos, diarreia, fraqueza, diplopia, visão turva, edema periférico, ataxia, labilidade emocional, confusão mental, amnésia, insônia, nervosismo, erupções cutâneas, alopécia, alteração do ciclo menstrual, dor abdominal, anorexia, dispepsia, aumento do apetite, constipação, ganho ou perda de peso, trombocitopenia, tremor, nistagmo, zumbido no ouvido, síndrome da influenza, rinite, eritema multiforme, hiperamonemia, hepatotoxicidade, pancreatite aguda, prolongamento do tempo de sangramento, aumento transitório das enzimas do fígado, parkinsonismo, encefalopatia, pseudoatrofia do cérebro e doenças hemostáticas, especialmente trombocitopenia $^{29}$.

12. VIGABATRINA: defeitos no campo visual, sedação, cefaleia, tontura, ataxia, transtornos de memória e comportamentais, parestesias, ganho de peso e tremor ${ }^{28}$.

Nota-se, pelo exposto anteriormente, que todos os medicamentos podem desencadear efeitos colaterais potencialmente incapacitantes, com destaque para aqueles relacionados às funções cognitivas e comportamentais, o que, contudo, deve ser analisado individualmente e de forma a relacionar o tipo, a intensidade e a distribuição ao longo do dia dos efeitos adversos em contraposição às características e exigências do trabalho do periciando.

O INSS $(2009)^{20}$, em documento que trata das diretrizes para tomada de decisão médico-pericial, sugere que o perito, em sua avaliação, busque sinais de intoxicação por drogas antiepiléticas como medida indireta de adesão ao tratamento e da gravidade do caso. Recomenda, também, que, em caso de suspeita de baixa adesão, o perito solicite dosagem sérica de droga antiepilética. A dosagem sérica não está disponível para todos os medicamentos e os valores de normalidade podem variar segundo os padrões de referência de cada laboratório, devendo o perito estar atento a tal aspecto.

Os casos de epilepsia refratária merecem especial atenção na avaliação médico-pericial ${ }^{13,20}$. Entretanto, a definição de epilepsia refratária a tratamento ainda não encontra consenso, considerando que a refratariedade a tratamento medicamentoso é conceito dinâmico na medida em que evoluem também os recursos farmacológicos.

Segundo Garzon $(2002)^{8}$,

"do ponto de vista teórico para que se pudesse considerar um paciente com epilepsia intratável de forma medicamentosa, o controle satisfatório das crises não poderia ser obtido com nenhuma das DAE (drogas antiepiléticas), usadas isoladamente ou em combinação, até doses ou niveis subtóxicos. Embora este conceito seja simples, a utilização de todas as DAE potencialmente eficazes, e em todas as combinações, $e$ praticamente impossivel em um determinado paciente uma vez que essa conduta demoraria mais de uma década".

Conclui-se não tratar-se de definição adequada para utilização na seleção de candidatos ao tratamento cirúrgico e, do mesmo modo, no cotidiano da prática pericial.

Para o Ministério da Saúde ${ }^{28}$, epilepsia refratária é aquela em que há "persistência de crises epiléticas apesar do uso de dois fármacos anticonvulsivantes de primeira linha, em doses adequadas". No contexto médicopericial, tal definição pode orientar as avaliações, mas deve ser relativizada, dadas as atuais opções terapêuticas existentes, incluindo os medicamentos disponíveis pelo Programa de Medicamentos Excepcionais do Ministério da Saúde, que tem como um dos critérios de inclusão de pacientes o enquadramento na definição de refratariedade descrita neste parágrafo.

Outra questão que se apresenta ao médico perito é a determinação do tempo de incapacidade em decorrência da necessidade de ajuste de doses de medicamentos. Não 
há como prever o tempo de tratamento da epilepsia, variando de caso a caso. Entretanto, o Ministério da Saúde do Brasil sugere que o tratamento seja revisado a cada três meses, prazo este que pode nortear o trabalho do perito em casos de indivíduos sem controle do quadro, em fase de ajuste de medicamentos, entre outros ${ }^{28}$.

No que se refere ao controle da síndrome,

"a maioria dos centros considera um periodo de tempo mínimo de 3 anos após a última crise associado à ausência de alterações paroxísticas ao eletroencefalograma para a suspensão do tratamento" 28 .

Somente após um período de cinco anos sem tratamento e sem crises, uma pessoa é considerada sem epilepsia ${ }^{2}$.

\section{Exames complementares e avaliação de incapacidade por epilepsia}

Há consenso de que o diagnóstico de epilepsia deve ser feito com base em dados clínicos ${ }^{3,20}$.

Entretanto, exames complementares podem auxiliar no diagnóstico, na identificação de lesões causadoras de epilepsia, na classificação do tipo de síndrome epilética e consequente escolha terapêutica e, também, no monitoramento da evolução do tratamento ${ }^{3}$.

O eletroencefalograma (EEG) é o exame mais utilizado na avaliação do paciente com epilepsia.

Há indicação de que o exame seja realizado em vigília e sono, considerando que sua sensibilidade é aumentada pela avaliação em sono ${ }^{3}$.

Segundo INSS (2009) $)^{20}$, o

"EEG pode muitas vezes ser interpretado de forma equivocada. Uma variante da normalidade pode facilmente ser confundida com atividade epileptiforme; indivíduos normais podem apresentar lentificações ou mesmo alterações epileptiformes sem qualquer significado clínico. Cerca de 0,5 a 1\% das pessoas normais, sem evidência clínica de epilepsia e sem outra doença cerebral apresentam anormalidades epileptiformes, localizadas ou generalizadas ao EEG. Por outro lado, indivíduos com epilepsia podem ter exame neurofisiológico completamente normal".

O vídeo-eletroencefalograma consiste do registro simultâneo de imagem do paciente e estudo eletroencefalográfico por 24 horas ao dia ao longo de vários dias e auxilia na identificação da localização da área epileptogênica ${ }^{3}$.

A tomografia computadorizada permite o diagnóstico de lesões estruturais do encéfalo, tais como malformações arteriovenosas, acidentes vasculares, lesões calcificadas e tumores do sistema nervoso central e identifica lesão associada à epilepsia em 30\% dos pacientes estudados, o que revela que a presença ou ausência isolada de lesões estruturais não pode ser encarada como sinônimo de doença não controlada ou incapacidade laborativa ${ }^{3}$.

A ressonância magnética também auxilia na identificação de lesões estruturais e recomenda-se que seja solicitada após estabelecimento de correlação eletroclínica ${ }^{3}$.

A Positron Emission Tomography (PET) é uma das formas de avaliar funcionalmente o encéfalo e pode detectar diferentes padrões no metabolismo cerebral da glicose nos períodos ictal e interictal ${ }^{3}$.

A Single Photon Emission Computed (SPECT) é outra forma de avaliar o encéfalo funcionalmente e revela padrões distintos de fluxo sanguíneo cerebral nos períodos ictal e interictal ${ }^{3}$.

A espectometria por ressonância magnética caracteriza-se pela pesquisa do marcador neuronal $\mathrm{N}$-acetilaspartato e pode auxiliar na localização da área epileptogênica ${ }^{3}$.

A ressonância magnética funcional é uma maneira não invasiva de identificar a área epileptogênica, além de estabelecer sua relação com outras áreas funcionais tais como as áreas motora, visual, da linguagem, da memória, etc ${ }^{3}$.

Observa-se que os exames complementares são úteis por permitir uma compreensão mais ampliada de cada caso, auxiliam na identificação de lesões estruturais relacionadas à epilepsia, das áreas epileptogênicas, apoiam as decisões terapêuticas. Contudo, devem ser examinados à luz da clínica do periciando e não há achados ou resultados específicos que sejam sinônimo de incapacidade laborativa quando analisados isoladamente.

\section{Ocupações e condições de trabalho específicas: incompatibilidade com a epilepsia?}

Sarmento \& Minayo-Gomez (2005) ${ }^{35}$, após modificação do proposto por Nierdermeyer (1990), apresentam lista de ocupações que devem ser evitadas pelos epiléticos. São elas: policiais, bombeiros, vigias solitários, instrutor de natação e salva-vidas, babás, profissionais de enfermagem, cirurgiões, motoristas profissionais, operadores de máquinas e/ou equipamentos, militares, profissionais que executam trabalhos em altura ou com uso de escadas. Stephen \& Brodie $(2008)^{36}$ apontam que a ausência de controle da epilepsia não é aceitável entre pessoas responsáveis por prestar cuidados a indivíduos vulneráveis.

Entretanto, os autores sugerem cautela na utilização dessa proposta, indicando sua aplicação integral apenas nos casos em que não haja completo controle do quadro. Para os demais, as restrições devem ser relativizadas ${ }^{35}$.

O documento que descreve as diretrizes para tomada de decisão médico-pericial do Instituto Nacional 
de Seguro Social recomenda encaminhamento precoce do segurado epilético à Reabilitação Profissional nos casos de profissões que envolvam atividades com riscos para si e para terceiros, tais quais piloto de avião, maquinista de trem, motorista de ônibus e caminhão, bombeiros, policiais, operadores de instrumentos e máquinas perigosas $^{20}$.

Em lugar de abordar tipos de ocupações, alguns autores propõem analisar as características e/ou condições em que a atividade é exercida para avaliar a possibilidade de inserção do epilético no trabalho e desaconselham a integração de epiléticos em atividades que envolvam: trabalho em altura, próximo de água, com exposição a alta voltagem e circuitos elétricos abertos, próximo ou sobre veículos em movimento, subterrâneo, em contato com fogo, fornos e fogões e em locais isolados ${ }^{21}$.

Novamente, o documento que descreve as diretrizes para tomada de decisão médico-pericial do Instituto Nacional de Seguro Social recomenda encaminhamento precoce do segurado epilético à Reabilitação Profissional em caso do exercício de atividades desenvolvidas em situação de risco permanente, tais quais trabalho em altura, contato com fogo, mergulho, etc ${ }^{20}$.

Verifica-se tendência dos autores em caracterizar incompatibilidade entre algumas ocupações ou situações específicas no trabalho e epilepsia, mesmo quando esta se encontra controlada.

\section{DISCUSSÃO}

Do exposto anteriormente, é possível apreender que a epilepsia é um problema de Saúde Pública e questão de interesse ao universo médico-pericial pela alta frequência com que indivíduos epiléticos se apresentam pleiteando o reconhecimento de incapacidade laborativa.

Seu diagnóstico por si só não é sinônimo de incapacidade e grande parte dos casos tem bom prognóstico com controle do quadro e preservação da capacidade laborativa. Entretanto, incapacidade laborativa pode advir dessa condição e, em geral, relaciona-se às suas repercussões somáticas ou psíquicas, comorbidades psiquiátricas, efeitos colaterais das drogas antiepiléticas ou quando se verifica risco à saúde do trabalhador e/ou a terceiros.

Considerando-se que a incapacidade é socialmente determinada e relaciona-se não somente aos aspectos biomédicos da doença, o perito deve avaliar cada caso em sua integralidade, ponderando fatores tais como acesso e características do sistema de saúde, grau de escolaridade do periciando, capacidade de adaptação a outras atividades laborais, entre outros.

Recomenda-se a utilização de várias ferramentas na construção da conclusão pericial: anamnese e exame físico detalhados, análise documental, entrevista com familiares ou acompanhantes, dosagem sérica de drogas antiepiléticas, quando necessário, e solicitação de documentação complementar (prontuário médico) nos casos de dúvidas.

Em suma, são fatores que favorecem a presença de incapacidade: crises frequentes, crises com perda de consciência, crises de início súbito, crises sem fatores desencadeantes, crises em vigília, não adesão ao tratamento, alcoolismo ou uso de outras drogas, epilepsia de causas estruturais, metabólicas ou desconhecidas, epilepsia refratária a tratamento, período de ajuste de doses de medicamentos.

Outros aspectos podem sinalizar gravidade da doença e devem ser observados pelo perito, embora haja que se relativizar sua aplicação como "determinantes" de incapacidade: acompanhamento em serviço de referência, inclusão em programas de dispensação de medicamentos especiais, realização de exames complementares diferenciados, indicação de avaliação neuropsicológica, politerapia em altas doses, internações frequentes, eletroencefalograma persistentemente alterado, indicação de tratamento cirúrgico.

No tocante aos efeitos do tratamento medicamentoso, verificou-se que todos os medicamentos estudados podem desencadear efeitos colaterais potencialmente incapacitantes, com destaque para aqueles relativos às funções cognitivas e comportamentais, o que, contudo, deve ser analisado individualmente e de forma a relacionar o tipo, a intensidade e a distribuição ao longo do dia dos efeitos adversos em contraposição às características e exigências do trabalho do periciando.

Os resultados de exames complementares são úteis por permitir uma compreensão mais ampliada de cada caso, mas devem ser examinados à luz da clínica do periciando e não há achados ou resultados específicos que sejam sinônimo de incapacidade laborativa quando analisados isoladamente.

Há tendência dos autores em caracterizar incompatibilidade entre algumas ocupações ou situações específicas no trabalho e epilepsia, mesmo quando esta se encontra controlada. São elas: profissões que envolvam atividades com riscos para si e para terceiros, tais quais piloto de avião, maquinista de trem, bombeiros, policiais, operadores de instrumentos e máquinas perigosas, vigias solitários, instrutor de natação e salva-vidas, babás, profissionais de enfermagem, cirurgiões, motoristas profissionais, militares, pessoas responsáveis por prestar cuidados a indivíduos vulneráveis, trabalho em altura ou com uso de escadas, próximo de água, com exposição a alta voltagem e circuitos elétricos abertos, próximo ou sobre veículos em movimento, subterrâneo, em contato com fogo, fornos e fogões.

\section{CONCLUSÕES}

Não há protocolos fechados sobre a avaliação de 
incapacidade laborativa por epilepsia. Contudo, existem condições norteadoras da avaliação médico-pericial.

Preparo técnico, avaliação individualizada, atenta e detalhada pelo perito favorecem a produção de prova técnica fortemente sustentada por conhecimento científico, alcançando-se assim o objetivo da perícia médica que é se fazer justiça.

Braga LC; Gianvecchio VAP. Specific conditions to help orient experts to characterize work disability caused by epilepsy. Saúde, Ética \& Justiça. 2014;19(2):67-77.

ABSTRACT: BACKGROUND: Epilepsy is a public health problem and an important cause of work disability in Brazil and worldwide and is a condition of interest for medical assessment. OBJECTIVE: To describe what conditions should be considered to help orient experts to characterize work disability caused by epilepsy. METHODS: Literature review. RESULTS AND DISCUSSION: The term diagnosis of epilepsy in itself is not synonymous with work disability. Most cases have a good prognosis with control, in which there is continued work capability. However, work disability can result from this condition and, in general, is related to somatic or psychological implications, psychiatric comorbidities, side effects of antiepileptic drugs and whenever there is risk to workers' health and/or other people. Factors related to disability are the following: frequent seizures, seizures with loss of consciousness, seizures of sudden onset without triggering factors, seizures in wakefulness, non-adherence to treatment, alcoholism or drug abuse, structural, metabolic or unknown causes of epilepsy, epilepsy resistant to treatment. Other aspects may indicate disease severity, but they should be considered in medical assessment in relation to the following: monitoring referral service, inclusion in programs for special drugs dispensation, complementary tests indication, indication of neuropsychological assessment, polytherapy in high doses, frequent interactions, persistently altered EEG, surgical treatment. All medications analyzed in this review may trigger potential side effects, especially those relating to cognitive and behavioral functions. The results of additional tests should be analyzed in association with symptoms and there are no findings or specific outcomes that are synonymous with work incapacity. Authors tend to characterize the incompatibility of certain occupations or labor situations with epilepsy, even when the disease is controlled. They are as follows: occupations involving activities with risks individuals themselves and others: airline pilots, train conductors, firemen, police officers, operators of hazardous machinery and instruments, solitary guards, swimming instructors and lifeguards, babysitters, nurses, surgeons, professional drivers, military, people responsible for providing care to disabled people, people working at heights or using stairs, near water, with exposure to high voltage electrical circuits, moving vehicles, underground or in contact with fire, furnaces and stoves. CONCLUSIONS: There are specific conditions that should be considered to help orient experts in characterizing work disability caused by epilepsy. Technical training, individualized evaluation, careful and detailed assessment all serve to guide expert judgment that is strongly supported by scientific knowledge, thereby achieving the goal of medical assessment: to see that justice is done.

KEY WORDS: Disabled persons; Epilepsy; Work capacity evaluation; Expert testimony.

\section{REFERÊNCIAS}

1. Fisher RS, van Emde Boas W, Blume W, Elger C, Genton P, Lee P, Engel J Jr. Epileptic seizures and epilepsy: definitions proposed by the International League Against Epilepsy (ILAE) and the International Bureau for Epilepsy (IBE). Epilepsia. 2005;46(4):470-2. DOI: 10.1111/j.00139580.2005.66104.x

2. World Health Organization. Global campaign against epilepsy. Geneva: WHO; 2000.

3. Yacubian EMT, Sakamoto AC. Epilepsia. In: Prado FC, Ramos J, do Valle JR. Atualização terapêutica: manual prático de diagnóstico e tratamento. São Paulo: Artes Médicas; 2007. p. 1120-7.

4. BERG AT, Berkovic SF, Brodie MJ, Buchhalter J, Cross JH, Boas WVE, Engel J, French J, et al. Terminologia e conceitos revistos para organização de crises e epilepsias. Relatório da Comissão da ILAE de Classificação e Terminologia, 20052009 [Internet]. 2010. [acesso em 06 dez. 2012]. Disponível em: http://www.ilae.org/visitors/centre/ctf/documents/ PortuguesefullarticleBergetal2010.pdf

5. Kwan P, Sander JW. The natural history of epilepsy: an epidemiological view. J Neurol Neurosurg Psychiatry. 2004;75(10):1376-81. DOI: 10.1136/jnnp.2004.045690

6. Angalakuditi M, Angalakuditi N. A comprehensive review of the literature on epilepsy in selected countries in emerging markets. Neuropysichiatr Dis Treat. 2011;7(1):585-97. DOI: 10.2147/NDT.S24966.

7. Sander JWAS, Sillanpää M. Natural history and prognosis. In: Engel-Junior J, Pedley TA. Epilepsy, a comprehensive textbook. Philadelphia: Lippincott-Raven; 1998.

8. Garzon E. Epilepsia refratária: conceito e contribuição das novas drogas antiepilépticas e de outras modalidades terapêuticas. Rev Neurociências. 2002;10(2): 66-82.

9. Sander JWAS, Schorvon SD. Incidence and prevalence studies in epilepsy and their methodological problems: a review. J Neurol Neurosurg Psychiatry. 1987;50(7):829-39.

10. Gracia F, deLao S, Castillo L, Larreategui M, Archbold C, Brenes M, Reeves WC. Epidemiology of epilepsy in Guaymi Indians from Bocas del Toro Province, Republic of Panama. Epilepsia. $\quad$ 1990;31(6):718-23. DOI: $\quad 10.1111 /$ j.15281157.1990.tb05512.x 
Braga LC; Gianvecchio VAP. Condições norteadoras para caracterização de incapacidade laborativa por epilepsia.

11. World Health Organization. Global campaign against epilepsy: out of shadows. Geneva: WHO; 2003.

12. Gomes MM. Epilepsia e incapacidade laborativa. J Epilepsy Clin Neurophysiol. 2009;15(3):130-4. DOI: http://dx.doi. org/10.1590/S1676-26492009000300007

13. Miziara CSMG, Miziara ID, Muñoz DR. Epilepsia e trabalho: quando a epilepsia deve ser considerada incapacitante? Saúde, Ética \& Justiça. 2011;16(2):103-10.

14. Gannon B, Nolan B. Disability and labour market participation. Ireland: Equalit Studies Unit; 2004.

15. Trevisol-Bittencourt PC, Ferreira MA, Marasciulo AC, Collares CF. Condições mais frequentes em um ambulatório de perícia neurológica. Arq Neuropsiquiatr. 2001;59(2-A):214-8. DOI: http://dx.doi.org/10.1590/S0004282X2001000200012

16. Aldenkamp AP. Effects of antiepileptic drugs on cognition. Epilepsia. 2001;42(Suppl 1):46-9. DOI: 10.1046/j.15281157.2001.00516.

17. Galluci Neto J, Marchetti RL. Aspectos epidemiológicos e relevância dos transtornos mentais associados à epilepsia. Rev Bras Psiquiatr. 2005;27(4):323-8. DOI: http://dx.doi. org/10.1590/S1516-44462005000400013

18. Wiebe S, Hesdorffer DC. Epilepsy: being ill in more ways than one. Epilepsy Curr. 2007;7(6):145-8. DOI: 10.1111/j.15357511.2007.00207.x

19. Tedrus GMAS, Fonseca LC, Oliveira EM, Fonseca ALA, Carneiro ACR, Carvalho RM. Factors associated with the employment situation and driving license of pacients with epilepsy. J Epilepsy Clin Neurophysiol. 2010;16(4):136-42. DOI: http://dx.doi.org/10.1590/S1676-26492010000400004

20. Brasil. Ministério da Previdência Social. Instituto Nacional Do Seguro Social. Diretrizes de apoio à decisão médicopericial em clínica médica. Parte I [Internet]. 2009. [acesso em $06 \mathrm{dez}$ 2012]. Disponível em: http://www.previdenciasocial. gov.br/arquivos/office/4_091021-153135-494.pdf

21. Miziara CSMG. Semiologia neurológica. In: Universidade Estadual Paulista. Coordenadoria de Saúde e Segurança do Trabalhador e Sustentabilidade Ambiental. Curso de extensão "Perícias em saúde". São Paulo: UNESP; 2011.

22. Loisel P, Durand MJ, Baril R, Gervais J, Falardeau M. Interorganizational collaboration in occupational rehabilitation: perceptions of an interdisciplinary rehabilitation team. J Occup Rehabil. 2005;15(4):581-90. DOI: $10.1007 / \mathrm{s} 10926-005-8036-\mathrm{x}$

23. Valente KDR, Thomé-Souza S, Kuczynski E, Negrão N. Depressão em crianças e adolescentes com epilepsia. Rev Psiquiatr Clín. 2004;31(6):290-9. DOI: http://dx.doi. org/10.1590/S0101-60832004000600004

24. Marchetti RL, Castro APW, Kurcgant D, Cremonese E, Galluci Neto J. Transtornos mentais associados à epilepsia. Rev Psiquiatr Clín. 2005;32(3):170-82.

25. Asadi-Pooya AA, Sperling MR. Depression and anxiety in patients with epilepsy, with or without other chronic disorders. Iran Red Crescent Med J. 2011;13(2):112-6.

26. Wannmacher L. Medicamentos que atuam sobre o sistema nervoso central. In: BRASIL. Ministério da Saúde. Formulário terapêutico nacional 2008. Rename 2006. Brasília: Ministério da Saúde; 2008. p. 539-55.

27. Brasil. Presidência da República. Ministério da Saúde. Portaria SAS/MS n 492 de 23 de setembro de 2010. Dispõe sobre protocolo clínico e diretrizes terapêuticas para tratamento da epilepsia. 2010. Diário Oficial da União, Brasília (DF). 201024 set.; Seção 1:673

28. Agência Nacional de Vigilância Sanitária. Fármacos utilizados no Sistema Nervoso Central [Internet]. 2012. [Acesso em $06 \mathrm{dez}$ 2012]. Disponível em: http://www. anvisa.gov.br/divulga/public/livro_eletronico/snc.html\#_ Toc25063616.

29. Brasil. Ministério da Saúde. Nota técnica $n^{\circ}$ 37/2012. Objetiva subsidiar a defesa da União em juízo e tornar mais acessível, aos operadores jurídicos em geral, informações de cunho técnico e científico, disponibilizadas em documentos oficiais produzidos pelos órgãos competentes do SUS e/ ou outras agências internacionais sobre o medicamento levetiracetam. 2012. [acesso em 06 dez 2012]. Disponível em: http://portalsaude.saude.gov.br/portalsaude/arquivos/ pdf/2012/Out/08/levetiracetam.pdf.

30. Brasil. Ministério da Saúde. Nota Técnica $n^{\circ}$ 62/2012. Objetiva subsidiar a defesa da União em juízo e tornar mais acessível, aos operadores jurídicos em geral, informações de cunho técnico e científico, disponibilizadas em documentos oficiais produzidos pelos órgãos competentes do SUS e/ ou outras agências internacionais sobre o medicamento oxcarbazepina. 2012. [acesso em 06 dez 2012]. Disponível em:http://portalsaude.saude.gov.br/portalsaude/arquivos/ pdf/2012/Out/05/oxcarbazepina.pdf.

31. Thome-Souza S, Valente KDR. Droga órfã: surgimento de um novo conceito. J. Epilepsy Clin. Neurophysiol. 2011;17(4):144-7. DOI: http://dx.doi.org/10.1590/S167626492011000400006

32. European Medicines Agency. Science Medicines Health. Inovelon [Internet]. 2011. [Acesso em 06 dez 2012]. Disponível em: http://www.ema.europa.eu/docs/en GB/ document_library/EPAR_-_Summary_for_the_public/ human/000660/WC500032938.pdf

33. Arroyo S. Estudio abierto com tiagabina em epilepsia parcial. Rev Neurol. 2000;31(8):728-32.

34. Sarmento MRS, Minayo-Gomez C. A epilepsia, o epilético e o trabalho: relações conflitantes. Cad Saúde Pública. 2000;16(1):183-93. DOI: http://dx.doi.org/10.1590/S0102311X2000000100019

35. Niedermeyer E. The Epilepsies: Diagnosis and Management. Baltimore: Urban \& Schwarzenberg; 1990.

36. Stephen LJ, Brodie MJ. Speccial problems: adults and elderly. Epilepsia. 2008;49(Suppl 1):45-9. DOI: 10.1111/j.15281167.2008.01450.x 